\title{
A Third-Order Boundary Condition for the Exterior Stokes Problem in Three Dimensions
}

\author{
By Georges H. Guirguis
}

\begin{abstract}
We approximate the Stokes operator on an exterior domain in three dimensions by a truncated problem on a finite subdomain. A third-order artificial boundary condition is introduced. We discuss the approximating behavior of the truncated problem and its discretization in a finite element space. Combined errors arising from truncation and discretization are considered.
\end{abstract}

1. Introduction. We study the numerical approximation to incompressible viscous flows in a domain exterior to a bounded star-shaped set in $\mathbb{R}^{3}$. In [10], [11] essential and natural boundary operators have been considered on finite subdomains of the exterior domain. It is our task in this paper to derive a higher-order, precisely a third-order, boundary condition. We extend the methods in [3], [10], [11], [12] to deal with the complexity of the governing vector equations in spherical polar coordinates. Let $\mathscr{Q}$ be a bounded star-shaped set in $\mathbb{R}^{3}$. Let $\Omega$ be the complement of its closure in $\mathbb{R}^{3}$. Let $x=\left(x_{1}, x_{2}, x_{3}\right)=(r, \theta, \varphi)$ denote a generic point in $\mathbb{R}^{3}$, and $|x|$ the distance from the origin, given by

$$
r=|x|=\left(x_{1}^{2}+x_{2}^{2}+x_{3}^{2}\right)^{1 / 2} \text {. }
$$

Let $\lambda=\left(\lambda_{1}, \lambda_{2}, \lambda_{3}\right)$ denote a multi-index and let $|\lambda|=\lambda_{1}+\lambda_{2}+\lambda_{3}$. Let

$$
D_{i} \equiv \frac{\partial}{\partial x_{i}}
$$

denote the distributional partial derivative with respect to the $i$ th coordinate and let

$$
D^{\lambda} \equiv D_{1}^{\lambda_{1}} D_{2}^{\lambda_{2}} D_{3}^{\lambda_{3}}
$$

Definition 1.1. For $m$ a nonnegative integer and $\alpha \in \mathbb{R}$ we define the weighted Sobolev space [13]

$$
W^{m, \alpha}(\Omega)=\left\{u \in D^{\prime}(\Omega): \int_{\Omega}\left(1+r^{2}\right)^{\alpha-m+|\lambda|}\left|D^{\lambda} u\right|^{2} d x<\infty,|\lambda| \leqslant m\right\},
$$

where $D^{\prime}(\Omega)$ denotes the space of distributions over $\Omega$.

Received June 2, 1986

1980 Mathematics Subject Classification (1985 Revision). Primary 35Q10, 65N15, 65N30. 
We refer the reader to [9], [10], [11], [13] for the properties of these spaces. $W^{m, \alpha}(\Omega)$ is a Hilbert space equipped with the inner product

$$
(u, v)_{m, \alpha ; \Omega}=\sum_{|\lambda| \leqslant m}\left\{\int_{\Omega}\left(1+r^{2}\right)^{\alpha-m+|\lambda|} D^{\lambda} u D^{\lambda} v d x\right\}
$$

and norm

$$
\|u\|_{m, \alpha ; \Omega}=\left[(u, u)_{m, \alpha ; \Omega}\right]^{1 / 2} .
$$

We let $W^{m-j-1 / 2}(\delta \Omega), j=1, \ldots, m-1$, denote the trace spaces on the boundary $\delta \Omega$, and we use the notation $\mathscr{W}^{m, \alpha}(\Omega)$ to denote the completion of $C_{0}^{\infty}(\Omega)$ in the $\|\cdot\|_{m, \alpha ; \Omega}$. We also use the notation of $W^{-m,-\alpha}(\Omega)$ to denote the dual spaces of $\stackrel{\circ}{W}^{m, \alpha}(\Omega)$ with duality pairing denoted by

$$
\langle u, v\rangle_{m, \alpha ; \Omega},
$$

equipped with the norm

$$
\|u\|_{-m,-\alpha ; \Omega}=\sup _{v \in \dot{W}^{m, \alpha}(\Omega)} \frac{\langle u, v\rangle_{m, \alpha ; \Omega}}{\|v\|_{m, \alpha, \Omega}} .
$$

The Stokes problem will be denoted in the sequel by the continuous problem as given by

$$
\begin{gathered}
-\Delta \mathbf{u}+\nabla p=\mathbf{f} \quad \text { in } \Omega, \\
\operatorname{div} \mathbf{u}=0 \quad \text { in } \Omega, \\
\left.\mathbf{u}\right|_{\delta \Omega}=0, \\
\lim _{|x| \rightarrow \infty} \mathbf{u}(x)=0,
\end{gathered}
$$

with the support of $f$ compact in $\Omega$. In [9], [11], problem (1.1)-(1.4) has been posed variationally on the weighted Sobolev spaces defined above. For the details of the proof we refer the reader to these references. Thus, we state the existence theorem.

THEOREM 1.2. The weak formulation of (1.1)-(1.4) is given by: Seek $(\mathbf{u}, p) \in$ $\left[\mathscr{W}^{1,0}(\Omega)\right]^{3} \times L^{2}(\Omega)$ such that

$$
\begin{gathered}
a(\mathbf{u}, \mathbf{v})+b(p, \mathbf{v})=f(\mathbf{v}) \quad \forall \mathbf{v} \in\left[\stackrel{\circ}{W}^{1,0}(\Omega)\right]^{3}, \\
b(q, \mathbf{u})=0 \quad \forall q \in L^{2}(\Omega),
\end{gathered}
$$

where

$$
\begin{gathered}
a(\mathbf{u}, \mathbf{v})=\int_{\Omega} \operatorname{grad} \mathbf{u}: \operatorname{grad} \mathbf{v} d x, \\
b(p, \mathbf{v})=-\int_{\Omega} p \operatorname{div} \mathbf{v} d x,
\end{gathered}
$$

has a unique solution pair $(\mathbf{u}, p)$ for $\mathbf{f} \in\left[W^{-1,0}(\Omega)\right]^{3}$, and there exists a constant $C>0$ such that

$$
\|\mathbf{u}\|_{1,0 ; \Omega}+\|p\|_{0,0 ; \Omega} \leqslant C\|\mathbf{f}\|_{-1,0 ; \Omega} .
$$

Furthermore, if $\Omega$ is a $C^{m}$ domain $[1]$ and $\mathbf{f} \in\left[W^{m-1, m}(\Omega)\right]^{3}$, then

$$
(\mathbf{u}, p) \in\left[W^{m+1, m}(\Omega) \cap \dot{\circ}^{1,0}(\Omega)\right]^{3} \times W^{m, m}(\Omega)
$$


and

$$
\|\mathbf{u}\|_{m+1, m ; \Omega}+\|p\|_{m, m ; \Omega} \leqslant C\|\mathbf{f}\|_{m-1, m ; \Omega} \text {. }
$$

It is essential, for computational purposes, to approximate the continuous problem by another problem, to be known in the sequel as the truncated problem, defined on a finite subdomain $\Omega_{R}$ of the original domain $\Omega$. Define the truncated domain to be

$$
\Omega_{R}=\Omega \cap B(0 ; R),
$$

where $B(0 ; R)$ denotes the sphere of radius $R$ centered at the origin. Let $\delta \Omega_{R}$ denote the large artificial boundary introduced to construct the truncated domain. Then the truncated problem is given by

$$
\begin{gathered}
-\Delta \mathbf{u}_{R}+\nabla p_{R}=\mathbf{f}_{R} \quad \text { in } \Omega_{R}, \\
\operatorname{div} \mathbf{u}_{R}=0 \quad \text { in } \Omega_{R}, \\
\mathbf{u}_{R}=0 \quad \text { on } \delta \Omega, \\
\beta\left(\mathbf{u}_{R}, p_{R}\right)=0 \quad \text { on } \delta \Omega_{R},
\end{gathered}
$$

where $\beta(\cdot, \cdot)$ denotes an artificial boundary operator needed at the large boundary $\delta \Omega_{R}$. It is important to point out at this stage that the use of higher-order boundary operators can only be associated to smoother solutions at the artificial boundary $\delta \Omega_{R}$, so that the artificial boundary condition at $\delta \Omega_{R}$ could be interpreted in the sense of trace spaces of Lions [15]. This is the case if $\mathbf{f}$ has a compact support in $\Omega_{R}$ or $\mathrm{f}$ is smooth enough for $R \geqslant R_{0}$. This is provided in Section 2.

In order to derive the approximating properties of the truncated problem, we will need the following estimates.

Proposition 1.3. Let $u \in C^{\infty}(\Omega) \cap W^{1,0}(\Omega)$ be such that $u=O\left(r^{-\alpha}\right)$ for $\alpha>1 / 2$ and $r \geqslant R$. Let $\Omega_{\infty}=\Omega \backslash \bar{\Omega}_{R}$ denote the open domain exterior to $\Omega_{R}$. Then we have the following:

$$
\begin{aligned}
\|u\|_{s, s-1 ; \Omega_{\infty}}=O\left(R^{-\alpha+1 / 2}\right), & s \geqslant 0, \\
\|u\|_{-s,-s+1 ; \Omega_{\infty}}=O\left(R^{-\alpha+1 / 2}\right), & s \geqslant 0, \\
\|u\|_{s-1 / 2, s-1 ; \delta \Omega_{R}}=O\left(R^{-\alpha+1 / 2}\right), & s \geqslant 1 / 2, \\
\|u\|_{-s+1 / 2,-s+1 ; \delta \Omega_{R}}=O\left(R^{-\alpha+1 / 2}\right), & s \geqslant 1 / 2 .
\end{aligned}
$$

Remark. As pointed out in [10], the result (1.11) can be shown for $s$ integer, then proceed by interpolation for any $s \geqslant 0$. Then, using a similar argument for the trace spaces, (1.13) can be shown. Finally, (1.12) and (1.14) can be shown by duality arguments. In the following sections we will derive the third-order boundary operator in spherical coordinates, obtain the variational formulation of "the truncated problem" and obtain error estimates relating the solutions of the truncated problem and the solution of the continuous problem on the domain $\Omega_{R}$. Finite element approximations of the truncated problem are discussed in detail in [10] and [11]; so we conclude the work with combined error estimates. 


\section{The Truncated Problem.}

2.1. Derivation of the Boundary Operator. Consider the Stokes operator in spherical coordinates in $\mathbb{R}^{3}$. Let

$$
\mathbf{u}=\left(u_{r}, u_{\theta}, u_{\varphi}\right) .
$$

The momentum equations are given by

$$
\begin{gathered}
-\Delta u_{r}-\frac{2 u_{r}}{r^{2}}-\frac{2}{r^{2} \sin \theta} \frac{\partial\left(u_{\theta} \sin \theta\right)}{\partial \theta}-\frac{2}{r^{2} \sin \theta} \frac{\partial u_{\varphi}}{\partial \varphi}+\frac{\partial p}{\partial r}=0, \\
-\Delta u_{\theta}+\frac{2}{r^{2}} \frac{\partial u_{r}}{\partial \theta}-\frac{u_{\theta}}{r^{2} \sin ^{2} \theta} \frac{2 \cos \theta}{r^{2} \sin ^{2} \theta} \frac{\partial u}{\partial \varphi}+\frac{1}{r} \frac{\partial p}{\partial \theta}=0, \\
-\Delta u_{\varphi}+\frac{2}{r^{2} \sin \theta} \frac{\partial u_{r}}{\partial \varphi}+\frac{2 \cos \theta}{r^{2} \sin ^{2} \theta} \frac{\partial u_{\theta}}{\partial \varphi}-\frac{u_{\varphi}}{r^{2} \sin ^{2} \theta}+\frac{1}{r \sin \theta} \frac{\partial p}{\partial \varphi}=0,
\end{gathered}
$$

and the continuity equation is given by

$$
\frac{1}{r^{2}} \frac{\partial\left(r^{2} u_{r}\right)}{\partial r}+\frac{1}{r \sin \theta} \frac{\partial\left(\sin \theta u_{\theta}\right)}{\partial \theta}+\frac{1}{r \sin \theta} \frac{\partial u_{\varphi}}{\partial \varphi}=0 .
$$

Using (2.4) and (2.1), we eliminate the presence of $u_{\theta}$ and $u_{\varphi}$ to get

$$
\frac{\partial p}{\partial r}=\Delta u_{r}+\frac{2}{r} \frac{\partial u_{r}}{\partial r}+\frac{2}{r^{2}} u_{r} .
$$

At this stage, it is essential to point out that Eq. (2.5) uncouples the velocity in the radial $r$ direction from the velocities in the tangential $\theta$ and $\varphi$ directions. It will be seen later that Eq. (2.5) will simplify the variational formulation of the problem.

Proposition 2.1. Let ( $(\mathbf{u}, p)$ denote the solution of the Stokes problem with support of $\mathbf{f}$ compact in $\mathbb{R}^{3}$ and $\operatorname{supp}(\mathbf{f}) \subset \subset B\left(0 ; R_{0}\right)$. For $R \geqslant R_{0}$ define

$$
\begin{gathered}
\beta_{1}\left(u_{r}, p\right)=p-\frac{\partial u_{r}}{\partial r}+\frac{r}{2}\left[\frac{\partial p}{\partial r}-\frac{\partial^{2} u_{r}}{\partial^{2} r}\right]-2 \frac{\partial u_{r}}{\partial r}-\frac{2}{r} u_{r}, \\
\beta_{2}\left(u_{\theta}\right)=\frac{\partial u_{\theta}}{\partial r}+\frac{1}{r} u_{\theta}, \\
\beta_{3}\left(u_{\varphi}\right)=\frac{\partial u_{\varphi}}{\partial r}+\frac{1}{r} u_{\varphi}, \\
\beta(\mathbf{u}, p)=\left[\begin{array}{c}
\beta_{1}\left(u_{r}, p\right) \\
\beta_{2}\left(u_{\theta}\right) \\
\beta_{3}\left(u_{\varphi}\right)
\end{array}\right] .
\end{gathered}
$$

Then,

$$
|\boldsymbol{\beta}(\mathbf{u}, p)|=O\left(R^{-3}\right) .
$$

Remark. The proof is a direct consequence of the asymptotic expansion of both the velocity and pressure fields [14]. Since conditions (2.7) and (2.8) are similar to the scalar case [12], we proceed also to give a brief explanation of the way the boundary operator (2.6) is generated. It is known [14] that the solution pair of the continuous Stokes problem in $\mathbb{R}^{3}$ satisfies

$$
(\mathbf{u}, p)=O\left(R^{-1}, R^{-2}\right),
$$


hence

$$
p-\frac{\partial u_{r}}{\partial r}=O\left(R^{-2}\right)
$$

and therefore

$$
p-\frac{\partial u_{r}}{\partial r}+\frac{r}{2}\left[\frac{\partial p}{\partial r}-\frac{\partial^{2} u_{r}}{\partial^{2} r}\right]=O\left(R^{-3}\right)
$$

Finally, the terms

$$
2 \frac{\partial u_{r}}{\partial r}+\frac{2}{r} u_{r}=O\left(R^{-3}\right)
$$

have been added for the sole purpose of eliminating the normal derivative of the radial velocity contribution at the large boundary $\delta \Omega_{R}$ in the variational formulation.

We simplify (2.6), using (2.5), to obtain

$$
\begin{aligned}
p-\frac{\partial u_{r}}{\partial r} & =-\frac{r}{2}\left[\frac{\partial p}{\partial r}-\frac{\partial^{2} u_{r}}{\partial r^{2}}\right]+2 \frac{\partial u_{r}}{\partial r}+\frac{2}{r} u_{r} \\
& =-\frac{r}{2}\left[\Delta u_{r}+\frac{2}{r} \frac{\partial u_{r}}{\partial r}+\frac{2}{r^{2}} u_{r}\right]+\frac{r}{2} \frac{\partial^{2} u_{r}}{\partial r^{2}}+2 \frac{\partial u_{r}}{\partial r}+\frac{2}{r} u_{r} \\
& =-\frac{1}{2 r \sin \theta} \frac{\partial}{\partial \theta}\left[\sin \theta\left(\frac{\partial u_{r}}{\partial \theta}\right)\right]-\frac{1}{2 r \sin ^{2} \theta} \frac{\partial^{2} u_{r}}{\partial \varphi^{2}}-\frac{1}{r} u_{r}
\end{aligned}
$$

Hence, the final form of the boundary operator in the radial direction is given by

$$
\begin{aligned}
\beta_{1}\left(u_{r}, p\right)= & p-\frac{\partial u_{r}}{\partial r} \\
& +\frac{1}{2 r \sin \theta} \frac{\partial}{\partial \theta}\left[\sin \theta\left(\frac{\partial u_{r}}{\partial \theta}\right)\right]+\frac{1}{2 r \sin ^{2} \theta} \frac{\partial^{2} u_{r}}{\partial \varphi^{2}}-\frac{1}{r} u_{r} .
\end{aligned}
$$

2.2. Variational Formulation of the Problem. Let $\left(\mathbf{u}_{R}, p_{R}\right)$ denote, respectively, the velocity and pressure solutions of the truncated problem. The velocity in spherical coordinates is given by

$$
\mathbf{u}_{R}=\left(u_{r}^{R}, u_{\theta}^{R}, u_{\varphi}^{R}\right) .
$$

Let $\left[W^{R}\left(\Omega_{R}\right)\right]^{3}$ denote the subspace of $\left[W^{1,0}\left(\Omega_{R}\right)\right]^{3}$ given by

$$
\left[W^{R}(\Omega)_{R}\right]^{3}=\left\{\left(u_{r}, u_{\theta}, u_{\varphi}\right) \in\left[W^{1,0}\left(\Omega_{R}\right)\right]^{3}, \mathbf{u}=0 \text { on } \delta \Omega, u_{r} \in W^{1,0}\left(\delta \Omega_{R}\right)\right\}
$$

equipped with the inner product

$$
\begin{aligned}
(\mathbf{u}, \mathbf{v})= & \int_{\Omega_{R}} \operatorname{grad} \mathbf{u}: \operatorname{grad} \mathbf{v} d x+\int_{\Omega_{R}} \frac{1}{\left(1+r^{2}\right)} \mathbf{u} \cdot \mathbf{v} d x \\
& +\int_{\delta \Omega_{R}} \frac{1}{\left(1+r^{2}\right)^{1 / 2}} \mathbf{u} \cdot \mathbf{v} d S+\int_{\delta \Omega_{R}}\left(1+r^{2}\right)^{1 / 2} \operatorname{grad}_{t} u_{r} \operatorname{grad}_{t} v_{r} d S \\
= & (\mathbf{u}, \mathbf{v})_{1,0 ; \Omega_{R}}+\left(u_{\theta}, v_{\theta}\right)_{0,-1 / 2 ; \delta \Omega_{R}}+\left(u_{\varphi}, v_{\varphi}\right)_{0,-1 / 2 ; \delta \Omega_{R}}+\left(u_{r}, v_{r}\right)_{1,1 / 2 ; \delta \Omega_{R}},
\end{aligned}
$$


with $\operatorname{grad}_{t}$ denoting a tangential derivative along the surface $\delta \Omega_{R}$. The norm generated by this inner product is given by

$$
\|\mathbf{u}\|_{R ; \Omega_{R}}=[(\mathbf{u}, \mathbf{u})]^{1 / 2}
$$

i.e.,

$$
\|\mathbf{u}\|_{R ; \Omega_{R}}^{2}=\|\mathbf{u}\|_{1,0 ; \Omega_{R}}^{2}+\left\|u_{\theta}\right\|_{0 ;-1 / 2 ; \delta \Omega_{R}}^{2}+\left\|u_{\varphi}\right\|_{0 ;-1 / 2 ; \delta \Omega_{R}}^{2}+\left\|u_{r}\right\|_{1,1 / 2 ; \delta \Omega_{R}}^{2} .
$$

Remark. Note that $\left[W^{R}\left(\Omega_{R}\right)\right]^{3}$ is not smooth enough to build $\left[W^{3 / 2,1 / 2}\left(\Omega_{R}\right)\right]^{3}$. In fact, we observe that

$$
\left[W^{3 / 2,1 / 2}\left(\Omega_{R}\right)\right]^{3} \subset\left[W^{R}\left(\Omega_{R}\right)\right]^{3} \subset\left[W^{1,0}\left(\Omega_{R}\right)\right]^{3} .
$$

We only require the smoothness of $u_{r}$ on the boundary $\delta \Omega_{R}$ in the sense of $W^{1,1 / 2}\left(\delta \Omega_{R}\right)$. Some of the properties of $\left[W^{R}\left(\Omega_{R}\right)\right]^{3}$ are listed in the following lemmas.

LEMMA 2.2. $\left[W^{R}\left(\Omega_{R}\right)\right]^{3}$ is a Hilbert space.

Let $\chi_{r}$ denote the characteristic function of the domain $\Omega_{R}$. Define the set $K$ to be

$$
K=\left\{u \mid u=\chi_{R} v, v \in C_{0}^{\infty}(\Omega)\right\}
$$

LEMMA 2.3. $K$ is dense in $\left[W^{R}\left(\Omega_{R}\right)\right]^{3}$.

The variational form of the momentum equation (1.7) can be obtained in the usual manner by multiplying by a smooth vector test function $\mathbf{v}$ and then integrating by parts to produce a form which, through continuity arguments, can be extended to build the weak formulation. This yields

$$
\begin{aligned}
& \int_{\Omega_{R}} \operatorname{grad} \mathbf{u}_{R}: \operatorname{grad} \mathbf{v} d x+\int_{\Omega_{R}} p_{R} \operatorname{div} \mathbf{v} d x \\
& \quad+\int_{\delta \Omega_{R}}\left(p_{R} \mathbf{n}-\mathbf{n} \cdot \operatorname{grad} \mathbf{u}_{R}\right) \cdot \mathbf{v} d s=\int_{\Omega_{R}} \mathbf{f} \cdot \mathbf{v} d x
\end{aligned}
$$

For $\delta \Omega_{R}$ the surface of a sphere, we have $\mathbf{n}=\mathbf{e}_{r}$. By using the expression for $\mathbf{n} \cdot \operatorname{grad} \mathbf{u}$ in spherical coordinates we can further simplify the integration on the boundary $\delta \Omega_{R}$ to

$$
\int_{\delta \Omega_{R}}\left(p_{R} \mathbf{n}-\mathbf{n} \cdot \operatorname{grad} \mathbf{u}_{R}\right) \cdot \mathbf{v} d S=\int_{\delta \Omega_{R}}\left(p_{R} v_{r}-v_{r} \frac{\partial u_{r}^{R}}{\partial r}-v_{\theta} \frac{\partial u_{\theta}^{R}}{\partial r}-v_{\varphi} \frac{\partial u_{\varphi}^{R}}{\partial r}\right) d S .
$$

Now, using the artificial boundary operator $\beta\left(\mathbf{u}_{R}, p_{R}\right)$, we get

$$
\begin{aligned}
\int_{\delta \Omega_{R}}\left(p_{R} \mathbf{n}-\mathbf{n} \cdot \operatorname{grad} \mathbf{u}_{R}\right) \cdot \mathbf{v} d S \\
=\int_{\delta \Omega_{R}}-\frac{v_{r}^{R}}{2 r \sin \theta} \frac{\partial}{\partial \theta}\left[\sin \theta \frac{\partial u_{r}^{R}}{\partial \theta}\right]-\frac{v_{r}}{2 r \sin ^{2} \theta} \frac{\partial^{2} u_{r}^{R}}{\partial \varphi^{2}} \\
\quad+\frac{u_{r}^{R} v_{r}+u_{\theta}^{R} v_{\theta}+u_{\varphi}^{R} v_{\varphi}}{r} r^{2} \sin \theta d \theta d \varphi
\end{aligned}
$$


Integrating by parts in the $\theta$ and the $\varphi$ variables over the surface of the sphere $\delta \Omega_{R}$, we obtain

$$
\begin{aligned}
\int_{\delta \Omega_{R}}\left(p_{R} \mathbf{n}-\mathbf{n} \cdot \operatorname{grad} \mathbf{u}_{R}\right) \cdot \mathbf{v} d S= & \int_{\delta \Omega_{R}} \frac{1}{2 r}\left[\frac{\partial u_{r}^{R}}{\partial \theta} \frac{\partial v_{r}}{\partial \theta}+\frac{1}{\sin ^{2} \theta} \frac{\partial u_{r}^{R}}{\partial \varphi} \frac{\partial v_{r}}{\partial \varphi}\right] \\
& +\frac{u_{r}^{R} v_{r}+u_{\theta}^{R} v_{\theta}+u_{\varphi}^{R} v_{\varphi}}{r} r^{2} \sin \theta d \theta d \varphi .
\end{aligned}
$$

Remark. Any distance $r$ on the boundary $\delta \Omega_{R}$ in the last expression can be replaced by $R$, since we are dealing with a sphere of radius $R$.

Define the bilinear form

$$
\begin{aligned}
A\left(\mathbf{u}_{R}, \mathbf{v}\right) & =\int_{\Omega_{R}} \operatorname{grad} \mathbf{u}_{R}: \operatorname{grad} \mathbf{v} d x \\
& =\int_{\delta \Omega_{R}} \frac{1}{2 r}\left[\frac{\partial u_{r}^{R}}{\partial \theta} \frac{\partial v_{r}}{\partial \theta}+\frac{1}{\sin ^{2} \theta} \frac{\partial u_{r}^{R}}{\partial \varphi} \frac{\partial v_{r}}{\partial \varphi}\right]+\frac{u_{r}^{R} v_{r}+u_{\theta}^{R} v_{\theta}+u_{\varphi}^{R} v_{\varphi}}{r} d S \\
& =\int_{\Omega_{R}} \operatorname{grad} \mathbf{u}_{R}: \operatorname{grad} \mathbf{v} d x+\frac{R}{2} \int_{\delta \Omega_{R}} \operatorname{grad}_{t} u_{r}^{R} \operatorname{grad}_{t} v d S+\frac{1}{R} \int_{\delta \Omega_{R}} \mathbf{u}^{R} \cdot \mathbf{v} d S
\end{aligned}
$$

Also define the bilinear form

$$
b(q, \mathbf{v})=-\int_{\Omega_{R}} q \operatorname{div} \mathbf{v} d x .
$$

Note that the forms $A(\cdot, \cdot)$ and $b(\cdot, \cdot)$ are continuous bilinear forms on $\left[W^{R}\left(\Omega_{R}\right)\right]^{3}$ $\times\left[W^{R}\left(\Omega_{R}\right)\right]^{3}$ and $L^{2}\left(\Omega_{R}\right) \times\left[W^{R}\left(\Omega_{R}\right)\right]^{3}$, respectively. We are now ready to state the weak formulation of the problem (1.7)-(1.10):

Seek $\left(\mathbf{u}_{R}, p_{R}\right) \in\left[W^{R}\left(\Omega_{R}\right)\right]^{3} \times L^{2}\left(\Omega_{R}\right)$ such that

$$
\begin{aligned}
A\left(\mathbf{u}_{R}, \mathbf{v}\right)+b\left(p_{R}, \mathbf{v}\right) & =(\mathbf{f}, \mathbf{v}) & & \forall \mathbf{v} \in\left[W^{R}\left(\Omega_{R}\right)\right]^{3}, \\
b\left(q, \mathbf{u}_{R}\right) & =0 & & \forall q \in L^{2}\left(\Omega_{R}\right) .
\end{aligned}
$$

The variational formulation is of the Brezzi type [3]. We need to verify the conditions for which a solution exists, namely the coercivity condition on $A(\cdot, \cdot)$ and the stability condition on $b(\cdot, \cdot)$. The coercivity of $A(\cdot, \cdot)$ is considered in the following lemma.

LEMMA 2.4. For $u \in\left[W^{1,0}\left(\Omega_{R}\right)\right]^{3}$ we have

$$
\int_{\Omega_{R}} \frac{u^{2}}{\left(1+r^{2}\right)} d x \leqslant C\left[\int_{\delta \Omega_{R}} \frac{u^{2}}{\left(1+r^{2}\right)^{1 / 2}} d S+\int_{\Omega_{R}}\left[\frac{\partial u}{\partial r}\right]^{2} d x\right] .
$$

Proof. See [10].

COROllary 2.5. The symmetric bilinear form $A(\cdot, \cdot)$ defined on $\left[W^{R}\left(\Omega_{R}\right)\right]^{3} \times$ $\left[W^{R}\left(\Omega_{R}\right)\right]^{3}$ is strongly coercive, i.e., there exists a constant $C$ independent of $R$ such that

$$
A\left(\mathbf{u}_{R}, \mathbf{u}_{R}\right) \geqslant C\left\|\mathbf{u}_{R}\right\|_{R ; \Omega_{R}}^{2} \quad \forall \mathbf{u}_{R} \in\left[W^{R}\left(\Omega_{R}\right)\right]^{3} .
$$


LEMMA 2.6. We have

$$
\sup _{v \in\left[W^{R}\left(\Omega_{R}\right)\right]^{3}} \frac{b(p, v)}{\|v\|_{R ; \Omega_{R}}} \geqslant C\|p\|_{0,0 ; \Omega_{R}} \quad \forall p \in L^{2}\left(\Omega_{R}\right) .
$$

Proof. We use the stability condition for the truncated problem with zero velocity boundary condition at the artificial interface $\Omega_{R}[10]$ :

$$
\sup _{v \in\left[W^{R}\left(\Omega_{R}\right)\right]^{3}} \frac{b(p, v)}{\|v\|_{R ; \Omega_{R}}} \geqslant \sup _{v \in\left[\dot{W}^{1.0}\left(\Omega_{R}\right)\right]^{3}} \frac{b(p, v)}{\|v\|_{R ; \Omega_{R}}} \geqslant C\|p\|_{0,0 ; \Omega_{R}} \quad \forall p \in L^{2}\left(\Omega_{R}\right) .
$$

We are now ready to state the following existence theorem concerning the variational formulation of the truncated problem.

THEOREM 2.7. Given $f_{R} \in\left[W^{-1,0}(\mathscr{Q})\right]^{3}$, with $\mathscr{Q} \subset \subset \Omega$, there exists a unique solution of problem (2.10)-(2.11). Furthermore, there exists a constant $C$ independent of $R$ such that

$$
\left\|\mathbf{u}_{R}\right\|_{R ; \Omega_{R}}+\left\|p_{R}\right\|_{0,0 ; \Omega_{R}} \leqslant C\|\mathbf{f}\|_{-1,0 ; \Omega_{R}}
$$

Now we are ready to consider the approximating behavior of the truncated problem.

2.3. Approximation Results. As mentioned earlier, an approximation that makes use of a higher-order artificial boundary operator will need a smoother solution of the continuous problem at the artificial boundary $\delta \Omega_{R}$. For the third-order boundary operator, we need the solution of the continuous problem to have the property

$$
u_{r} \in W^{1,1 / 2}\left(\delta \Omega_{R}\right)
$$

Let $\mathbf{e}_{R}=\mathbf{u}-\mathbf{u}_{R}$ and $\mu_{R}=p-p_{R}$ denote the error in the velocity and the pressure, respectively. Then the pair $\left(\mathbf{e}_{R}, \mu_{R}\right)$ satisfies

$$
\begin{gathered}
-\Delta \mathbf{e}_{R}+\nabla \mu_{R}=\mathbf{0} \quad \text { in } \Omega_{R}, \\
\operatorname{div} \mathbf{e}_{R}=0 \quad \text { in } \Omega_{R}, \\
\mathbf{e}_{R}=0 \quad \text { on } \delta \Omega, \\
\beta\left(\mathbf{e}_{R}, \mu_{R}\right)=\beta_{2}(\mathbf{u}, p) \quad \text { on } \delta \Omega_{R} .
\end{gathered}
$$

The problem of estimating the pair $\left(\mathbf{e}_{R}, \mu_{R}\right)$ can be formulated variationally as follows:

Seek $\left(\mathbf{e}_{R}, \mu_{R}\right) \in\left[W^{R}\left(\Omega_{R}\right)\right]^{3} \times L^{2}\left(\Omega_{R}\right)$ such that

$$
\begin{gathered}
A\left(\mathbf{e}_{R}, \mathbf{v}\right)+b\left(\mu_{R}, \mathbf{v}\right)=\langle\beta(\mathbf{u}, p) \cdot \mathbf{v}\rangle_{1 / 2,0 ; \delta \Omega_{R}} \quad \forall \mathbf{v} \in\left[W^{R}\left(\Omega_{R}\right)\right]^{3} \\
b\left(q, \mathbf{e}_{R}\right)=0 \quad \forall q \in L^{2}\left(\Omega_{R}\right)
\end{gathered}
$$

where the expression in the right-hand side can be written as

$$
\begin{aligned}
\langle\beta(\mathbf{u}, p) \cdot \mathbf{v}\rangle_{1 / 2,0 ; \delta \Omega_{R}}= & -\langle p-\mathbf{n} \cdot \operatorname{grad} \mathbf{u}, \mathbf{v}\rangle_{1 / 2,0 ; \delta \Omega_{R}}+\frac{1}{2}\left(u_{r}, v_{r}\right)_{1,1 / 2 ; \delta \Omega_{R}} \\
& +\frac{1}{2}\left(u_{r}, v_{r}\right)_{1,1 / 2 ; \delta \Omega_{R}}+\left(u_{\theta}, v_{\theta}\right)_{0, \pm 1 / 2 ; \delta \Omega_{R}}\left(u_{\varphi}, v_{\varphi}\right)_{0,-1 / 2 ; \delta \Omega_{R}} .
\end{aligned}
$$

Thus we see that the quantity $\beta(\mathbf{u}, p)$ is in $\left[W^{-1 / 2,0}\left(\delta \Omega_{R}\right)\right]^{3}$ in the sense of Lions 
[15]. Now, similar to Theorem 2.7 , we have

THEOREM 2.8. (i) The variational form (2.17)-(2.18) has a unique solution pair $\left(\mathbf{e}_{R}, \mu_{R}\right)$, and there exists a constant $C$ independent of $R$ such that

$$
\left\|\mathbf{e}_{R}\right\|_{R ; \Omega_{R}}+\left\|\mu_{R}\right\|_{0,0 ; \Omega_{R}} \leqslant C\|\beta(\mathbf{u}, p)\|_{-1 / 2,0 ; \delta \Omega_{R}} .
$$

(ii) For supp(f) compact in $\Omega$ we have

$$
\left\|\mathbf{e}_{R}\right\|_{R ; \Omega_{R}}+\left\|\mu_{R}\right\|_{0,0 ; \Omega_{R}} \leqslant \frac{C}{R^{2.5}}
$$

where again the constant is independent of $R$.

Proof. To prove (i), we use Theorem 2.7 with right-hand side given by

$$
\langle\beta(\mathbf{u}, p), v\rangle_{1 / 2,0 ; \delta \Omega_{R}} \text {. }
$$

As for (ii), this follows from (i) and Proposition 1.3.

\section{Discretization of the Truncated Problem.}

3.1. The Discrete Problem. We use notation similar to [7]. Let

$$
\bar{\Omega}_{R}=\bigcup_{K \in \tau_{h}} K
$$

where $K$ denotes a simplex in $R^{3}$. Let $h(K)$ be defined to be the maximum length of an edge belonging to the simplex $K$ and let $h$ be defined to be

$$
h=\max _{K \in \tau_{h}} h(K) \text {. }
$$

In [10], [11] it has been shown that the finite element approximation in the weighted spaces has qualitative properties similar to the results obtained for the Stokes problem in bounded domains. We refer the reader to the references [2], [5], [6], [7] for proper choices of stable pairs of finite element spaces for the velocity and pressure, respectively. Let $V_{h}$ and $S_{h}$ denote finite-dimensional subspaces [ $\left.W^{R}\left(\Omega_{R}\right)\right]^{3}$ and $L^{2}\left(\Omega_{R}\right)$, respectively. Let $T_{h}$ denote the trace space of $V_{h}$ on $\delta \Omega_{R}$. Define $Z_{h}$ to be the null space associated with the form $b(\cdot, \cdot)$, given by

$$
Z_{h}=\left\{\mathbf{v}_{r h} \in V_{h} \mid b\left(q_{r h}, \mathbf{v}_{r h}\right)=0 \forall q_{r h} \in S_{h}\right\} .
$$

The variational form of the discrete problem is now given by:

Seek $\left(\mathbf{u}_{r h}, p_{r h}\right) \in V_{h} \times S_{h}$ such that

$$
\begin{gathered}
A\left(\mathbf{u}_{r h}, \mathbf{v}_{r h}\right)+b\left(p_{r h}, \mathbf{v}_{r h}\right)=\mathbf{f}_{r h}\left(\mathbf{v}_{r h}\right) \quad \forall \mathbf{v}_{r h} \in V_{h}, \\
b\left(q_{r h}, \mathbf{u}_{r h}\right)=0 \quad \forall q_{r h} \in S_{h} .
\end{gathered}
$$

The following assumptions can be verified for a variety of finite element spaces for the pressure and velocity:

(A1) There exists a map, denoted by

$$
r_{h} \in \mathscr{L}\left(\left[W^{2,1}\left(\Omega_{R}\right)\right]^{3} \cap V ; Z_{h}\right),
$$

and a positive integer $\nu$ such that

with

$$
b\left(q_{R h}, \operatorname{div} r_{h} \mathbf{v}-\mathbf{v}\right)=0 \quad \forall q_{R h} \in S_{h}
$$

$$
\left\|\mathbf{v}-r_{h} \mathbf{v}\right\|_{1,0 ; \Omega_{R}} \leqslant C h^{m}\|v\|_{m+1, m ; \Omega_{R}}
$$

and $1 \leqslant m \leqslant \nu$. 
(A2) There exists an orthogonal projection operator

$$
s_{h} \in \mathscr{L}\left(\left[W^{2,3 / 2}\left(\delta \Omega_{R}\right)\right] ; T_{h}\right)
$$

and a positive integer $\nu$ such that

$$
\left\|v-s_{h} v\right\|_{1,1 / 2 ; \delta \Omega_{R}} \leqslant C h^{m}\|v\|_{m+1, m+1 / 2 ; \delta \Omega_{R}} \quad \forall v \in W^{m+1, m+1 / 2}\left(\delta \Omega_{R}\right),
$$

with $1 \leqslant m \leqslant \nu$.

(A3) The orthogonal projection denoted by

$$
\rho_{h} \in \mathscr{L}\left(W^{m, m}\left(\Omega_{R}\right) \cap S ; S_{h}\right)
$$

satisfies

$$
\left\|q-\rho_{h} q\right\|_{0,0 ; \Omega_{R}} \leqslant C h^{m}\|v\|_{m, m ; \Omega_{R}} \quad \forall q \in W^{m, m}\left(\Omega_{R}\right) \cap S .
$$

(A4) For every $q_{R h} \in S_{h}$ there exists a $\mathbf{w}_{R h} \in V_{h}$ such that

$$
\left(q_{R h}-\operatorname{div}_{h} \mathbf{w}_{R h}, s_{h}\right)=0 \quad \forall s_{h} \in S_{h}
$$

with

$$
\left\|\mathbf{w}_{R h}\right\|_{1,0 ; \Omega_{R}} \leqslant C\left\|q_{R h}\right\|_{0,0 ; \Omega_{R}} .
$$

Remark. The assumptions (A1, A3, A4) were needed for lower-order artificial boundary operators and have been discussed in [10], [11]. Also, Assumption (A2) can be easily verified using the methods in [10], [11] and then the isomorphism between $W^{m+1, \alpha}\left(\delta \Omega_{R}\right)$ and $W^{m+1, \beta}\left(\delta \Omega_{R}\right)$ [13]. We can now state the following theorem.

THEOREM 3.1. Under the hypotheses (A1), (A2), (A3) and (A4), problem (3.1)-(3.2) has exactly one solution pair $\left(\mathbf{u}_{R h}, p_{R h}\right) \in V_{h} \times S_{h}$, and

$$
\lim _{h \rightarrow 0}\left\{\left\|\mathbf{u}_{R h}-\mathbf{u}_{R}\right\|_{1,0 ; \Omega_{R}}+\left\|p_{R h}-p_{R}\right\|_{0,0 ; \Omega_{R}}\right\}=0 \text {. }
$$

Moreover, if the pair

$$
\left(\mathbf{u}_{R}, p_{R}\right) \in\left\{W^{s+1, s}\left(\Omega_{R}\right) \cap\left[W^{R}\left(\Omega_{R}\right)\right]^{3}\right\} \times\left\{W^{s, s}\left(\Omega_{R}\right)\right\},
$$

we have the usual bound

$$
\left\{\left\|\mathbf{u}_{R h}-\mathbf{u}_{R}\right\|_{1,0 ; \Omega_{R}}+\left\|p_{R h}-p_{R}\right\|_{0,0 ; \Omega_{R}}\right\} \leqslant C h^{m}\left\{\left\|\mathbf{u}_{R}\right\|_{m+1, m ; \Omega_{R}}+\left\|p_{R}\right\|_{m, m ; \Omega_{R}}\right\}
$$

for $1 \leqslant m \leqslant s$ and $C$ independent of $h$ and $R$.

3.2. Combined Error Estimates. As in [10], [11], we now combine the error estimates due to truncation and discretization.

THEOREM 3.2. There exists a constant $C$ independent of $h$ and $R$ such that

$$
\left\{\left\|\mathbf{u}_{R h}-\mathbf{u}\right\|_{1,0 ; \Omega_{R}}+\left\|p_{R h}-p\right\|_{0,0 ; \Omega_{R}}\right\} \leqslant C\left\{h^{m}+R^{-2.5}\right\},
$$

with $m$ depending on the choice of the finite element spaces used in the discretization of the truncated problem.

Finally, it is essential to point out for two-parameter approximations that the balance between the truncation error and the discretization error should be maintained for the optimality of the approximation [8]. As a consequence, larger simplices can be used in the far field, instead of using a quasiuniform mesh, and thus achieving the same accuracy with a lower number of degrees of freedom. 
Department of Mathematics

North Carolina State University

Raleigh, North Carolina 27695-8205

1. R. A. Adams, Sobolev Spaces, Academic Press, New York, 1975.

2. J. Boland, Finite Element and the Divergence Constraint for Viscous Flow, Ph.D. thesis, CarnegieMellon University, 1983.

3. F. BREZZI, "On the existence, uniqueness and approximation of saddle-point problems arising from Lagrangian multipliers,” RAIRO Anal. Numér. Sér. Rouge, v. 8, 1974, pp. 129-151.

4. M. CANTOR, "Numerical treatment of potential type equations on $R^{n}$ : Theoretical considerations," SIAM J. Numer. Anal., v. 20, 1983, pp. 72-85.

5. P. Ciarlet, The Finite Element Method for Elliptic Problems, North-Holland, Amsterdam, 1978.

6. M. Crouzeix \& P. Raviart, “Conforming and non-conforming finite element methods for solving the stationary Stokes equation," RAIRO Anal. Numér. Sér. Rouge, v. 7, 1973, pp. 33-75.

7. V. Girault \& P. Raviart, Finite Element Approximation of the Navier-Stokes Equations, Lecture Notes in Math., vol. 749, Springer-Verlag, Berlin and New York, 1979.

8. C. Goldstein, "The finite element method with non-uniform mesh sizes for unbounded domains," Math. Comp., v. 36, 1981, pp. 387-404.

9. G. H. Guirguis, "On the existence, uniqueness and regularity of the exterior Stokes problem in $R^{3}$," Comm. Partial Differential Equations, v. 11, 1986, pp. 567-594.

10. G. H. Guirguis \& M. D. Gunzburger, "On the approximation of the exterior Stokes problem in three dimensions," RAIRO Anal. Numér. (To appear.)

11. G. H. Guirguis, On the Existence, Uniqueness, Regularity and Approximation of the Exterior Stokes Problem in $R^{3}$, Ph.D. Thesis, University of Tennessee, Knoxville, 1983.

12. Alvin Bayliss, Max Gunzburger \& Eli Turkel, "Boundary conditions for the numerical solution of elliptic equations in exterior regions," SIAM J. Appl. Math., v. 42, 1982, pp. 430-451.

13. B. Hanouzet, "Espaces de Sobolev avec poids. Application a un problème de Dirichlet dans un demi-espace," Rend. Sem. Mat. Univ. Padova, v. 46, 1971, pp. 227-272.

14. O. LADYZHENSKaya, The Mathematical Theory of Viscous Incompressible Flow, Gordon and Breach, New York, 1969.

15. J. L. Lions \& E. Magenes, Non-Homogeneous Boundary Value Problems and Applications, Springer-Verlag, New York, 1972. 